\title{
ID16B Beamline at the ESRF: a Nanoprobe for the Characterization of Nanomaterials and Nanodevices
}

\author{
J. Segura-Ruiz ${ }^{1}$, R. Tucoulou ${ }^{1}$, J. Villanova ${ }^{1}$, D. Salomon ${ }^{1}$, J. Eymery ${ }^{2}$ and G. Martinez-Criado ${ }^{3}$ \\ ${ }^{1}$ (ESRF - The European Synchrotron) \\ Grenoble, France \\ jaime.segura@esrf.fr \\ ${ }^{2}$ Univ. Grenoble Alpes, CEA, INAC \\ Grenoble, France \\ ${ }^{3}$ Instituto de Ciencia de Materiales de Madrid (ICMM) \\ Madrid, Spain
}

\section{Extended Abstract}

Nanomaterials and nano-devices are continuously evolving. This continuous evolution requires powerful characterization techniques to better understand and further improve the properties of the materials and the performances of the devices. The advanced characterization tools provided by synchrotron installations can play an important role in these R\&D processes. Here, the compositional and optical properties of NW-based InGaN coaxial nano-Light Emitting Diodes will be studied using the ID16B hard X-ray nanoprobe beamline.

The ID16B beamline at the European Synchrotron Radiation Facility (ESRF) [1] is a nano-probe that provides a focused (down to $50 \times 50 \mathrm{~nm}^{2}$ ), intense (up to $10^{12} \mathrm{ph} / \mathrm{s}$ ), hard X-rays (up to $33 \mathrm{keV}$ ) beam. ID16B offers several characterization techniques and diverse sample environments. In this study we will use X-ray fluorescence (XRF) and X-ray excited optical luminescence (XEOL) simultaneously to characterize at Liquid-He temperature InGaN/GaN coaxial nano-LEDs. The coaxial LED heterostructure was fabricated by metalorganic vapour phase epitaxy and consists of an inner n-doped GaN core ( $\sim 2$ $\mu \mathrm{m}$ diameter) with five periods of $\operatorname{~n}_{x} \mathrm{Ga}_{1-x} \mathrm{~N} / \mathrm{GaN}$ MQWs shell (1.25-nm-thick InGaN well with $\sim 15 \%$ In concentration and 10-nm-thick GaN barrier) [2]. The nano-LEDs were dispersed on a Si substrate and pre-localized with the help of a Scanning Electron Microscope. The Si-substrate was then mounted on a liquid-He mini-cryostat compatible with the experimental setup available on the beamline ID16B and cooled down to $6 \mathrm{~K}$.

The sample was scanned with the $50 \times 50 \mathrm{~nm}^{2}$ beam and the XRF and and XEOL signals were collected simultaneously. Both, XRF and XEOL signals were analysed using the PyMCA software [3] in order to obtain elemental and emission correlated maps. The combined use of XRF and XEOL techniques with the He-cryostat available on ID16B provided unique space-resolved information to clarify the interplay between the compositional and optical properties of these nano-devices at the nanometre scale. The sharpness of the emission features observed in the XEOL spectra thanks to the very low temperatures provided by the He-cryostat allowed the visualization nanometre sized regions of the device having very localized emission with different peak energies. This emission-energy- allowed filtering was used to localize quantum-dot like regions within In-rich regions at the bottom of the coaxial LEDs, in the area where the deposition of the InGaN/GaN MQWs shell was not completed. The direct comparison between the XRF and the XEOL maps allowed to attribute this emission to InGaN nuclei with different In-concentrations in the range between $x=0.01$ to $x=0.4$. These results demonstrate the power of ID16B as a characterization tool for the study of complex nanomaterials and single nano-devices.

\section{References}

[1] G. Martínez-Criado, J. Villanova, R. Tucoulou, D. Salomon, J.-P. Suuronen, S. Labouré, C. Guilloud, V. Valls, R. Barrett, E. Gagliardini, Y. Dabin, R. Baker, S. Bohic, C. Cohen and J. Morse, "ID16B: a hard X-ray nanoprobe beamline at the ESRF for nano-analysis," J. Synchrotron Rad, vol. 23, p. 344, 2016.

[2] Robert Koester, Jun-Seok Hwang, Damien Salomon, Xiaojun Chen, Catherine Bougerol, Jean-Paul Barnes, Daniel Le Si Dang, Lorenzo Rigutti, Andres de Luna Bugallo, Gwénolé Jacopin, Maria Tchernycheva, Christophe Durand, Joël Eymery, "M-Plane Core-Shell InGaN/GaN Multiple-Quantum-Wells on GaN Wires for Electroluminescent Devices," Nano Lett., vol. 11, p. 4839, 2011. 
[3] V.A. Solé, E. Papillon, M. Cotte, Ph. Walter, J. Susini, "A multiplatform code for the analysis of energy-dispersive Xray fluorescence spectra," J. Spectrochim., Act A, Part B, vol. 62, p. 63, 2007. 